\title{
MICROCLIMA EM VINHEDOS DE 'NIAGARA ROSADA' EM DIFERENTES SISTEMAS DE CONDUÇÃO DURANTE SAFRAS DE INVERNO E DE VERÃO ${ }^{1}$
}

\author{
MÁRIO JOSÉ PEDRO JÚNIOR², JOSÉ LUIZ HERNANDES ${ }^{3}$, \\ GABRIEL CONSTANTINO BLAIN ${ }^{4}$, GLAUCO DE SOUZA ROLIM ${ }^{5}$
}

RESUMO - Na região vitícola de Jundiaí, o tradicional sistema de condução das plantas em espaldeira vem sendo paulatinamente substituído pela manjedoura na forma de Y. Alguns produtores têm lançado mão do uso de cobertura plástica para conferir maior proteção aos cachos de uva. Como esses diferentes sistemas de condução provocam alterações no microclima dos vinhedos, foram feitas medições de radiação solar global, umidade relativa, temperaturas máxima e mínima do ar no ambiente externo e na altura dos cachos de vinhedos de 'Niagara Rosada' conduzidos em espaldeira a céu aberto e em Y, com e sem cobertura de plástico, durante o período das safras de inverno e de verão. Verificou-se, que durante a safra de verão, as diferenças entre os valores diários de radiação solar, temperatura máxima do ar e umidade relativa foram mais elevadas em relação às da safra de inverno na comparação entre os sistemas de condução e o ambiente externo. Os vinhedos conduzidos em espaldeira apresentaram menores valores de radiação solar e umidade relativa do ar, na altura dos cachos. As temperaturas máximas e mínimas do ar foram semelhantes em todos os sistemas de condução e durante as diferentes safras. O uso de cobertura plástica no sistema de condução em Y não influiu na umidade relativa do ar e na temperatura mínima, porém propiciou aumento nos valores de temperatura máxima do ar.

Termos de indexação: uva de mesa, cultivo protegido, cobertura plástica.

\section{MICROCLIMATE IN 'NIAGARA ROSADA' VINEYARDS UNDER DIFFERENT TRELLIS SYSTEMS DURING THE WINTER AND THE SUMMER CROPS}

\begin{abstract}
At the grapevine growing region of Jundiaí (State of São Paulo - Brazil) the trellis system with single unilateral cordon and vertical upward branches is the most used by the growers. Nowadays it is been gradually replaced by the Y shaped trellis system. Still, some producers have been using plastic overhead cover for protection of the grape clusters. The different trellis systems cause modifications in the microclimate of the vineyard, so measurements of daily values of solar radiation, relative humidity, maximum and minimum air temperatures were taken in the external environment and at cluster height in 'Niagara Rosada' vineyards conducted in vertical with upward branches trellis system under open sky conditions and $\mathrm{Y}$ shaped trellis system, with and without plastic overhead cover, during the winter and the summer crops. During the summer growing season the differences in daily values of solar radiation, maximum air temperature and relative humidity obtained at cluster height and at external conditions were higher when compared to the winter crop for the different trellising systems evaluated. The vines in the vertical trellis system showed lower values of solar radiation and relative humidity at cluster height when compared to the Y shaped system. The values of maximum and minimum temperatures were similar in the vertical trellis and $\mathrm{Y}$ shaped system, during the winter and the summer crop. The plastic overhead cover on the Y shaped trellis did not influence the values of relative humidity and minimum temperature, however, resulted higher values of maximum air temperature.
\end{abstract}

Index terms: table grape, solar radiation, temperature, relative humidity, plastic overhead cover, trellis system.

\footnotetext{
1(Trabalho 179-12). Recebido em: 06-06-2012. Aceito para publicação em: 06-11-2012.

${ }^{2}$ Eng. Agr. Dr., PqC Centro de Ecofisiologia e Biofísica, IAC, Campinas-SP, Brasil, e-mail: mpedro@iac.sp.gov.br Bolsista do CNPq. ${ }^{3}$ Biólogo MS., PqC Centro de Fruticultura, IAC, Jundiaí-SP, Brasil. E-mail: jlhernandes@iac.sp.gov.br

${ }^{4}$ Eng. Agrícola Dr., PqC Centro de Ecofisiologia e Biofísica, IAC, Campinas-SP, Brasil. E-mail: gabriel@iac.sp.gov.br

${ }^{5}$ Eng. Agr. Dr., PqC Centro de Ecofisiologia e Biofísica, IAC, Campinas-SP, Brasil. E-mail: glaucorolim@gmail.com
} 


\section{INTRODUÇÃO}

A viticultura da região de Jundiaí (SP) está baseada na produção da uva para mesa 'Niagara Rosada' visando ao consumo in natura. As videiras são tradicionalmente cultivadas durante a safra de verão a céu aberto, sendo o sistema de condução em espaldeira com cordão esporonado único o mais utilizado. Porém, atualmente, o viticultor, visando à obtenção de maior renda, tem lançado mão de novas alternativas tecnológicas, como o cultivo em duas safras por ano (verão e inverno); diferentes sistemas de condução e, também, uso de cobertura plástica para a proteção das videiras (CHAVARRIA et al., 2007; PEDRO JÚNIOR et al., 2011).

A utilização do sistema de condução em manjedoura, na forma de $\mathrm{Y}$, está em crescente uso pelos viticultores paulistas. As vantagens do sistema de condução em Y residem na facilidade dos tratos culturais,no manuseio dos cachos e na melhoria da qualidade do produto, além de permitir melhor exposição das folhas e cachos à radiação solar. Esse sistema de condução tem sido utilizado com sucesso também no Rio Grande do Sul, em uvas para vinho, por facilitar a utilização de cobertura de plástico para a proteção das videiras, propiciando aumento de produção e por minimizar o uso de fungicidas no controle de doenças fúngicas (CHAVARRIA et al., 2007; MOTA et al., 2008; CARDOSO et al., 2008; CHAVARRIA et al., 2009; PEDRO JÚNIOR et al., 2011).

De acordo com Ferreira et al. (2004),Pedro Júnior et al. (2006),Pedro Júnior et al. (2007), Cardoso et al. (2008) e Chavarria et al. (2009), modificações no sistema de condução da videira, aliado ao cultivo protegido, induzem alterações no microclima do vinhedo. As principais alterações no microclima de vinhedos conduzidos sob cobertura de plástico ou telado plástico têm sido relatadas em respeito à radiação solar e à radiação fotossinteticamente ativa, temperatura do ar e umidade relativa do ar (FERREIRA et al., 2004; LULU et al., 2006; CARDOSO et al., 2008; CHAVARRIA et al., 2009; CONCEIÇÃO; MARIN, 2009; CARDOSO et al., 2010; PEDRO JÚNIOR et al., 2011; COMIRAN et al., 2012).

A radiação solar disponível às videiras é reduzida pela cobertura plástica em, aproximadamente, 15 a 30\% em relação à radiação externa (CARDOSO et al., 2008). Também a radiação solar fotossinteticamente ativa é diminuída no interior de ambientes protegidos (CARDOSO et al., 2008; CARDOSO et al., 2010). A atenuação da radiação solar é atribuída ao ângulo da cobertura em relação ao sol, à espessura e à composição do plástico, e aos fatores inerentes à sua degradação e ao depósito de resíduos (CHAVARRIA; SANTOS, 2009).

$\mathrm{O}$ ambiente condicionado no vinhedo, sob cultivo protegido, é devido à mudança no balanço de energia que traz consequências na temperatura do ar, principalmente com relação às máximas (CHAVARRIA; SANTOS, 2009). Temperaturas máximas mais elevadas $\left(1 \mathrm{a} 3^{\circ} \mathrm{C}\right)$ no interior de vinhedos conduzidos sob cobertura plástica, em relação às externas, foram relatadas por Ferreira et al. (2004), em Jundiaí (SP), para 'Cabernet Sauvignon'; Cardoso et al. (2008) e Chavarria et al. (2009), em Flores da Cunha (RS), para 'Moscato Giallo'. Com respeito às temperaturas mínimas relatadas na literatura, têm sido pequenas as diferenças entre cultivo protegido e ambiente externo (FERREIRA et al., 2004; CHAVARRIA et al., 2009; CONCEIÇÃO; MARIN, 2009; PEDRO JÚNIOR et al., 2011). No caso da umidade relativa, as diferenças entre os vinhedos sob ambiente protegido e o ambiente externo também não têm apresentado valor elevado (FERREIRA et al., 2004; CARDOSO et al., 2008; CHAVARRIA et al., 2009; PEDRO JÚNIOR et al., 2011), provavelmente, em função de terem sido as medições feitas em ambientes com abertura nas laterais, permitindo circulação do ar entre as plantas.

Considerando as alterações microclimáticas que ocorrem nos vinhedos, resultante dos diferentes sistemas de condução e do uso de cobertura plástica, foi desenvolvido este experimento na região produtora de 'Niagara Rosada' em Jundiaí (SP), visando a caracterizar as diferenças provocadas na temperatura do ar, umidade relativa e radiação solar nos sistemas de condução em espaldeira e em manjedoura na forma de Y com e sem cobertura de plástico, durante as safras de inverno e de verão.

\section{MATERIAL E MÉTODOS}

O experimento foi desenvolvido em vinhedo existente em área experimental do Instituto Agronômico de Campinas (IAC/APTA/SAA), localizada em

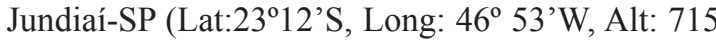
$\mathrm{m})$. No local, predominam os relevos ondulado e fortemente ondulado, com declividade acima de $12 \%$. O clima, de acordo com a classificação de Köppen, é $\mathrm{Cfa}$ para as regiões mais baixas e $\mathrm{Cfb}$ para as mais altas. As observações microclimáticas foram feitas durante agosto de 2008 a junho de 2009. A cultivar utilizada foi a 'Niagara Rosada', que é uma mutação somática da 'Niagara Branca', encontrada em 1933 na região de Louveira, e constitui- se na principal cultivar na região. As plantas foram enxertadas sobre IAC 766 'Campinas', adaptado às condições ambientais paulistas. 
O vinhedo, no espaçamento de $2 \times 1 \mathrm{~m}$,foi conduzido nos sistemas espaldeira e manjedoura na forma de $\mathrm{Y}$, com e sem cobertura de plástico. O sistema de condução em espaldeira com cordão esporonado simples é um sistema de condução de baixo custo de implantação, no qual os ramos da videira são dispostos na forma vertical (tipo renque). O sistema de condução em manjedoura em forma de ' $Y$ ' foi utilizado em videiras conduzidas a céu aberto e com cobertura de plástico (filme de polietileno de baixa densidade de 150 micras). Tratos culturais, como capina, remoção do broto apical e laterais (desponte e desnetamento), adubação e controle de doenças fúngicas foram feitos seguindo as práticas recomendadas para a cultura. As podas curtas foram realizadas em época normal (segunda quinzena de agosto), para a safra de verão, e extemporânea (primeira quinzena de fevereiro), para a safra de inverno. Foi feita aplicação de Dormex ${ }^{\circledR}$ a $4 \%$ para garantir a uniformidade da brotação.

As condições microclimáticas nos diferentes sistemas de condução foram avaliadas durante o período de maior enfolhamento das videiras (55 a 125 dias após a poda). A radiação solar global, temperatura e umidade relativa do ar foram determinadas por meio de medições com sistema automático de aquisição de dados (Datalogger CR23x, da Campbell Scientific) com leituras a cada minuto, para o registro dos valores diários, tendo sido utilizados os seguintes sensores:

a) radiação solar global - sensor CS300-L (Apogee PYR-P Pyranometer) instalado externamente (acima do dossel) e tubos solarímetros (TSL, Delta $T$ Devices) instalados abaixo do dossel, na altura dos cachos.

b) temperatura do ar e umidade relativa sensores tipo HMP45C-L (Campbell Scientific) instalados em microabrigos situados na altura dos cachos do vinhedo.

Os dados diários de temperatura máxima e mínima e de umidade relativa do ar do ambiente externo foram obtidos em posto meteorológico do Instituto Agronômico de Campinas (IAC/APTA/ SAA-SP), situado nas proximidades do vinhedo. As diferenças entre os valores médios ou totais diários dos parâmetros microclimáticos obtidos nos diferentes tratamentos (ambiente externo; sistemas de condução e uso de cobertura plástica) foram comparadas por meio do teste $t$, para amostras, presumindo variâncias equivalentes (comprovada pelo teste F), ao nível de $5 \%$.

\section{RESULTADOS E DISCUSSÃO}

Na Figura 1, é apresentada a variação da temperatura do ar máxima e mínima, da umidade relativa do ar e da radiação solar em vinhedos de Niagara Rosada durante o período compreendido entre 55 e 125 dias após a poda (período de maior enfolhamento das videiras). São também apresentadas comparações entre valores médios diários de radiação solar global e da umidade relativa do ar (Tabela 1). A comparação entre os valores das temperaturas máxima e mínima do ar obtidos em ambiente externo e na altura dos cachos, para todos os sistemas de condução durante as safras de inverno e de verão, pode ser verificada na Tabela 2. Na Figura 2, é apresentada a variação diária da radiação solar, temperatura e umidade relativa do ar em dias considerados ensolarados para as safras de inverno e de verão.

Na Tabela 1, considerando-se o período de maior enfolhamento das videiras, pode-se observar que a diferença entre os valores médios de radiação solar global, para os diferentes ambientes (tratamentos), tanto para a safra de inverno quanto para a de verão, mostrou-se significativa ao nível de $5 \%$,pelo teste t. As maiores diferenças entre os valores de radiação solar global medidos na altura dos cachos dos diferentes sistemas de condução e no ambiente externo foram obtidas para a espaldeira, durante a safra de inverno $\left(10,6 \mathrm{MJ} \cdot \mathrm{m}^{-2} \cdot \mathrm{dia}^{-1}\right)$ e de verão $\left(14,6 \mathrm{MJ} . \mathrm{m}^{-2} \cdot \mathrm{dia}^{-1}\right)$. Em seguida o sistema de condução em Y com e sem cobertura de plástico para a safra de verão, respectivamente com 11,0 e $13,7 \mathrm{MJ} \cdot \mathrm{m}^{-2} \cdot \mathrm{dia}^{-1}$. Durante a safra de verão, as diferenças foram maiores que na safra de inverno, provavelmente devido aos maiores valores de radiação solar que naturalmente ocorrem na época do verão, em comparação à de inverno.

$\mathrm{Na}$ comparação entre diferentes sistemas de condução, verificou-se que a radiação solar medida na altura dos cachos foi menor no sistema em espaldeira que no Y, em ambas as safras. Essas diferenças podem ser visualizadas na variação diária da radiação solar (Figura 2), em dias ensolarados, para cada uma das safras analisadas. A disposição aproximadamente horizontal dos ramos no sistema em Y permite que maior quantidade de radiação solar chegue aos cachos em comparação aos ramos na vertical do sistema em espaldeira, para o qual maior quantidade de folhas, na vertical, diminui a radiação solar disponível na altura dos cachos. A utilização de cobertura plástica, cuja transmissividade foi cerca de $20 \%$, influiu na quantidade de radiação solar disponível na altura dos cachos, tendo sido as maiores 
diferenças observadas na safra de verão $\left(2,7 \mathrm{MJ} . \mathrm{m}^{-2}\right.$. $\left.\mathrm{dia}^{-1}\right)$ em relação à safra de inverno $\left(1,5 \mathrm{MJ} \cdot \mathrm{m}^{-2} \cdot \mathrm{dia}^{-1}\right)$.

Em relação à umidade relativa média diária (Figuras 1c e 1d e Tabela 1), foi observado que, para todos os tratamentos, os valores medidos na altura dos cachos foram superiores ao ambiente externo. Essa diferença foi da ordem de 3\% para a espaldeira e $5 \%$ para o Y. Ao comparar os valores de umidade relativa entre os sistemas de condução, observou-se serem inferiores em aproximadamente 2,7\% na espaldeira, em relação ao Y sem cobertura de plástico. Também foi observado que a colocação de cobertura plástica no sistema em Y não provocou diferenças nos valores de umidade relativa, tanto durante a safra de inverno quanto na de verão. Esse fato também foi observado por Lulu et al. (2006) em videiras 'Romana' cultivadas sob cobertura de plástico; por Chavarria et al. (2007) em vinhedo de 'Moscato Giallo' conduzido em Y sob cobertura de plástico, e por Conceição e Marin (2009) em parreiral de BRS Morena sob tela plástica de polietileno.

Em relação às temperaturas máximas (Figuras 1a e 1b e Tabela 2), medidas ao nível do cacho no sistema de condução em espaldeira, verificou-se que foram superiores às do ambiente externo em $1,8^{\circ} \mathrm{C}$ e $2,4^{\circ} \mathrm{C}$, respectivamente, para as safras de inverno e verão. Para o sistema de condução em Y sem cobertura plástica, os valores das temperaturas máximas durante a safra de inverno e de verão também foram superiores aos do ambiente externo em cerca de $1,9^{\circ} \mathrm{C}$ (Tabela 2). Essas diferenças foram semelhantes às relatadas por Comiran et al. (2012) para 'Niagara Rosada' conduzida em latada, na região de Bento GonçalvesRS. De maneira geral, durante o período da safra de verão, em relação ao de inverno, foram observadas maiores diferenças entre as temperaturas máximas nos diferentes sistemas de condução, em comparação às do ambiente externo.

Ao se comparar os sistemas de condução em espaldeira e Y sem cobertura de plástico, não foram observadas diferenças estatísticas nos valores da temperatura máxima, pois como pode ser visto na Tabela 2, as diferenças variaram entre 0,1 e $0,5^{\circ} \mathrm{C}$ para as diferentes safras. A utilização de cobertura de plástico no sistema em Y permitiu verificar que as temperaturas máximas foram mais elevadas que no ambiente externo em $2,9^{\circ} \mathrm{C}$, para a safra de inverno, e $2,6^{\circ} \mathrm{C}$, para a de verão. Também, as temperaturas máximas no $\mathrm{Y}$ com plástico foram mais elevadas que nas plantas sem proteção, durante a safra de inverno, em $1,0^{\circ} \mathrm{C}$, enquanto para a safra de verão as diferenças foram de $0,8^{\circ} \mathrm{C}$. Esse aumento nos valores de temperatura máxima, devido ao uso da referida proteção, também foi verificado por Ferreira et al. (2004), Chavarria et al. (2007)e Cardoso et al. (2008) em vinhedos cultivados sob cobertura plástica.

No caso das temperaturas mínimas (Tabela

2) medidas ao nível dos cachos, tanto para o sistema em espaldeira quanto para o Y, foi observado que as diferenças com o ambiente externo não foram estatisticamente significativas durante a safra de inverno e de verão. Apenas para sistema em Y com cobertura de plástico foram observadas temperaturas mínimas superiores ao ambiente externo, tendo sido $1,0^{\circ} \mathrm{C}$ para a safra de verão e $0,8^{\circ} \mathrm{C}$ para a de inverno. As diferenças entre as temperaturas mínimas, tanto durante a safra de verão quanto na de inverno, não foram significativas quando comparados os sistemas de condução e o ambiente externo. Esse padrão de comportamento da temperatura mínima está de acordo com resultados apresentados por Chavarria et al. (2007) e Chavarria et al. (2009) em vinhedos de 'Moscato Giallo' sob cobertura de plástico.

Nas Figuras 2c,d pode ser verificado que as diferenças entre os tratamentos foi menor durante o período noturno (das 19 às 7 horas) em comparação ao período diurno (das 8 às 18 horas), quando a temperatura do ar ao nível dos cachos foi superior ao ambiente externo.

De maneira geral, as alterações microclimáticas foram maiores durante a safra de inverno em comparação à de verão, e o uso de cobertura de plástico influenciou principalmente na radiação solar e na temperatura máxima do ar quando comparadas ao ambiente externo. A comparação da influência exercida pelo sistema de condução mostrou diferenças na umidade relativa e da radiação solar, tendo sido obtidos maiores valores no sistema de condução em manjedoura na forma de $\mathrm{Y}$, em relação ao sistema em espaldeira. 


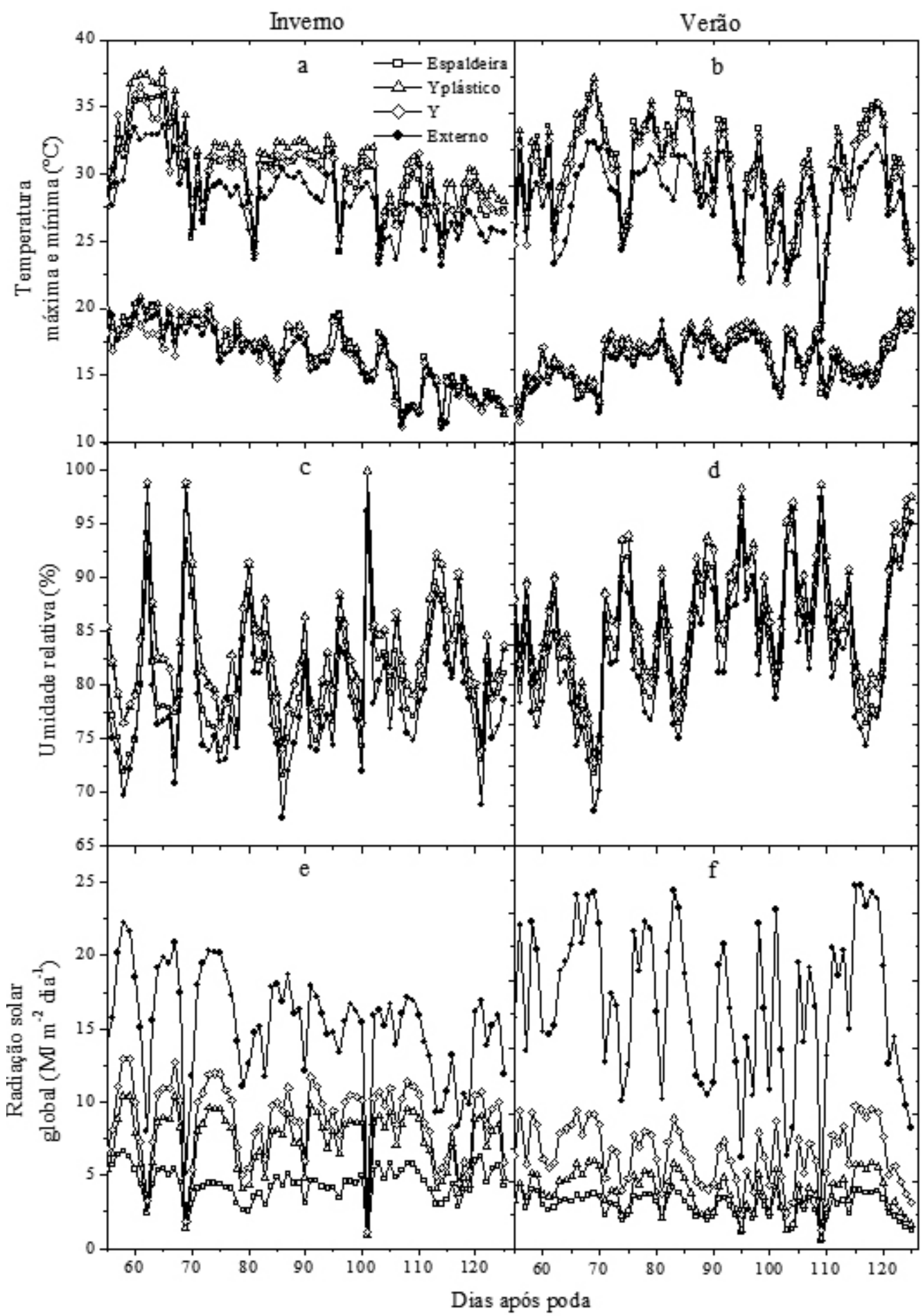

FIGURA 1-Valores médios diários da temperatura máxima e mínima do ar (a, b); umidade relativa (c, d) e radiação solar global (e,f) durante o período das safras de inverno e de verão, medidos em condições de posto meteorológico e na altura dos cachos, em vinhedos de 'Niagara Rosada' conduzidos em espaldeira e manjedoura em Y, sem e com cobertura de plástico. 

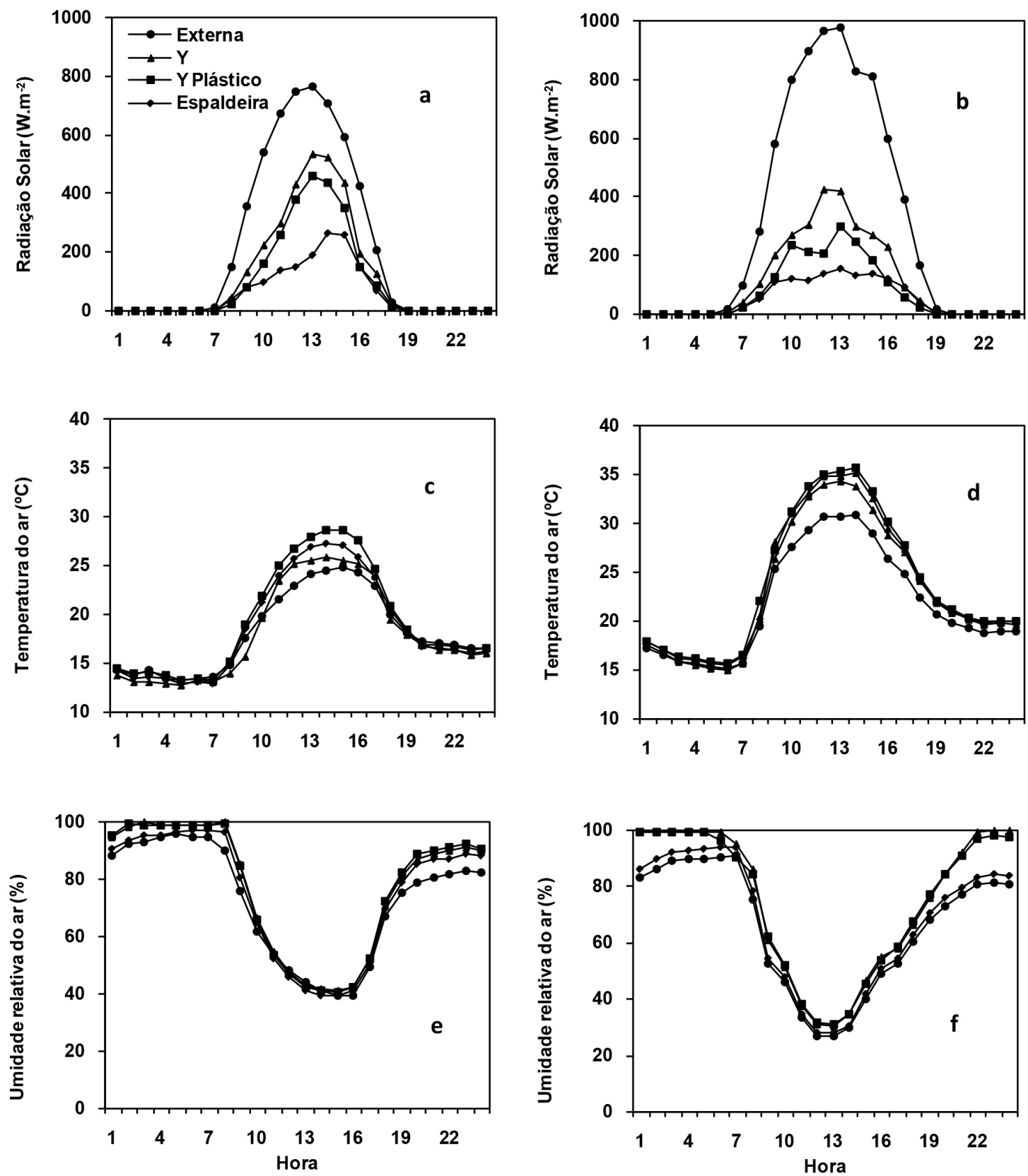

FIGURA 2- Variação diária da radiação solar, temperatura e umidade relativa do ar no ambiente externo e ao nível dos cachos de videiras 'Niagara Rosada', conduzidas em espaldeira a céu aberto e em manjedoura na forma de Y, com e sem cobertura de plástico, em dia ensolarado, durante as safras de inverno (esquerda) e de verão (direita). 
TABELA 1- Diferenças entre valores médios diários de radiação solar global e umidade relativa do ar medidos em ambiente externo e na altura dos cachos de videiras conduzidas em espaldeira e em manjedoura na forma de Y, com e sem cobertura de plástico, durante as safras de inverno e de verão em Jundiaí (SP).

\begin{tabular}{|c|c|c|c|c|c|c|c|}
\hline \multirow[t]{2}{*}{ Variável } & \multirow{2}{*}{$\begin{array}{l}\text { Comparação entre } \\
\text { tratamentos }\end{array}$} & \multicolumn{3}{|c|}{ Safra de inverno } & \multicolumn{3}{|r|}{ Safra de verão } \\
\hline & & DT & NS & CVT & DT & NS & CVT \\
\hline \multirow{5}{*}{$\begin{array}{r}\text { Radiação Solar } \\
\left(\mathrm{MJ} . \mathrm{m}^{-2} \cdot \mathrm{dia}^{-1}\right)\end{array}$} & Espaldeira x Externo & 10,6 & $*$ & Espaldeira $<$ Externo & 14,6 & $*$ & Espaldeira $<$ Externo \\
\hline & Y x Externo & 6,1 & $*$ & $\mathrm{Y}<$ Externo & 11,0 & $*$ & $\mathrm{Y}<$ Externo \\
\hline & Y plástico $\mathrm{x}$ Externo & 7,6 & $*$ & Y plástico $<$ Externo & 13,7 & $*$ & Y plástico $<$ Externo \\
\hline & Espaldeira x Y & 4,5 & $*$ & Espaldeira $<Y$ & 3,6 & $*$ & Espaldeira $<$ Y \\
\hline & Y plástico $\mathrm{x} Y$ & 1,5 & $*$ & Y plástico $<Y$ & 2,7 & $*$ & $\mathrm{Y}$ plástico $<\mathrm{Y}$ \\
\hline \multirow{5}{*}{$\begin{array}{l}\text { Umidade } \\
\text { relativa do ar } \\
\qquad(\%)\end{array}$} & Espaldeira x Externo & 2,0 & $*$ & Espaldeira $>$ Externo & 2,5 & $*$ & Espaldeira $>$ Externo \\
\hline & Y x Externo & 4,8 & $*$ & $\mathrm{Y}>$ Externo & 4,9 & $*$ & $\mathrm{Y}>$ Externo \\
\hline & Y plástico x Externo & 4,9 & * & Y plástico $>$ Externo & 5,5 & $*$ & Y plástico $>$ Externo \\
\hline & Espaldeira x Y & 3,0 & $*$ & Espaldeira $<$ Y & 2,4 & $*$ & Espaldeira < Y \\
\hline & Y plástico $\mathrm{x} Y$ & 0,1 & ns & Y plástico $=Y$ & 0,6 & $\mathrm{~ns}$ & Y plástico $=$ Y \\
\hline
\end{tabular}

DT = diferença entre tratamentos; NS = nível de significância; CVT = comparação dos valores médios entre tratamentos; ns = não significativo; *= significativo ao nível de $5 \%$.

TABELA 2 - Diferenças entre valores médios diários de temperatura máxima e mínima do ar medidos em ambiente externo e na altura dos cachos de videiras conduzidas em espaldeira e em manjedoura na forma de $\mathrm{Y}$, com e sem cobertura de plástico, durante as safras de inverno e de verão em Jundiaí (SP).

\begin{tabular}{|c|c|c|c|c|c|c|}
\hline \multirow[t]{2}{*}{ Variável } & \multirow{2}{*}{$\begin{array}{l}\text { Comparação entre } \\
\text { tratamentos }\end{array}$} & \multicolumn{3}{|c|}{ Safra de inverno } & \multicolumn{2}{|r|}{ Safra de verão } \\
\hline & & DT & NS & CVT & DT NS & CVT \\
\hline \multirow{5}{*}{$\begin{array}{l}\text { Temperatura } \\
\text { máxima } \\
\left({ }^{\circ} \mathrm{C}\right)\end{array}$} & Espaldeira x Externo & 1,8 & $*$ & Espaldeira $>$ Externo & 2,4 & Espaldeira $>$ Externo \\
\hline & Y x Externo & 1,9 & $*$ & $\mathrm{Y}>$ Externo & 1,8 & $\mathrm{Y}>$ Externo \\
\hline & Y plástico x Externo & 2,9 & $*$ & Y plástico $>$ Externo & $2,6 *$ & Y plástico $>$ Externo \\
\hline & Espaldeira X Y & 0,1 & ns & Espaldeira $=Y$ & $0,5 \mathrm{~ns}$ & Espaldeira $=Y$ \\
\hline & Y plástico x Y & 1,0 & $*$ & Y plástico $>Y$ & $0,8 *$ & Y plástico $>Y$ \\
\hline \multirow{5}{*}{$\begin{array}{l}\text { Temperatura } \\
\text { mínima } \\
\left({ }^{\circ} \mathrm{C}\right)\end{array}$} & Espaldeira x Externo & 0,5 & ns & Espaldeira $=$ Externo & $0,2 \mathrm{~ns}$ & Espaldeira $=$ Externo \\
\hline & Y x Externo & 0,2 & ns & $\mathrm{Y}=$ Externo & $0,6 \mathrm{~ns}$ & $\mathrm{Y}=$ Externo \\
\hline & Y plástico x Externo & 0,7 & $*$ & Y plástico $>$ Externo & $0,8 *$ & Y plástico $>$ Externo \\
\hline & Espaldeira x Y & 0,3 & ns & Espaldeira $=Y$ & $0,4 \mathrm{~ns}$ & Espaldeira $=\mathrm{Y}$ \\
\hline & Y plástico x Y & 0,5 & ns & Y plástico $=$ Y & $0,2 \mathrm{~ns}$ & Y plástico $=$ Y \\
\hline
\end{tabular}

DT = diferença entre tratamentos; NS = nível de significância; CVT = comparação dos valores médios entre tratamentos; ns = não significativo; $*=$ significativo ao nível de $5 \%$.

\section{CONCLUSÕES}

1- As diferenças entre os valores diários de radiação solar, temperatura máxima e umidade relativa obtidos nos diferentes sistemas de condução e os do ambiente externo foram maiores durante a safra de verão em comparação à de inverno.
2-Os vinhedos com sistema de condução em espaldeira apresentam menores valores de radiação solar e umidade relativa do ar em relação aos conduzidos no sistema de manjedoura na forma de Y.

3-As temperaturas máximas e mínimas do ar foram semelhantes, nos sistemas de condução em espaldeira e em Y, tanto durante a safra de inverno quanto na de verão. 
4-O uso de cobertura plástica no vinhedo com sistema de condução em $\mathrm{Y}$ não influiu na umidade relativa do ar e na temperatura mínima, porém propiciou aumento nos valores da temperatura máxima do ar.

\section{REFERÊNCIAS}

CARDOSO, L.S.; BERGAMASCHI, H.; COMIRAN, F.; CHAVARRIA, G.; MARODIN, G.A.B.; DALMAGO, G.A.; SANTOS, H.P.; MANDELLI, F. Alterações micrometeorológicas em vinhedos pelo uso de coberturas de plástico. Pesquisa Agropecuária Brasileira, Brasília, v.43, n.4, p. 441-447, 2008.

CARDOSO, L.S.; BERGAMASCHI, H.; COMIRAM, F.; CHAVARRIA, G.; MARODIN, G.A.B; DALMAGO, G.A.; SANTOS, H.P.; MANDELLI, F. Padrões de interceptação de radiação solar em vinhedos com e sem cobertura plástica. Revista Brasileira de Fruticultura, Jaboticabal, v.32, p.161171, 2010.

CHAVARRIA, G.; SANTOS, H.P.; Manejo de videiras sob cultivo protegido. Ciência Rural, Santa Maria, v.39, n.6, p.1917-1924, 2009.

CHAVARRIA, G.; CARDOSO, L.S.; BERGAMASCHI, H.; SANTOS, H.P.; MANDELLI, F.; MARODIN, G.A.B. Microclima de vinhedos sob cultivo protegido. Ciência Rural, Santa Maria, v.39, n.7, p. 2029-2034, 2009.

CHAVARRIA, G.; SANTOS, H.P.; SÔNEGO, O.R.; MARODIN, G.A.B.; BERGAMASCHI, H.; CARDOSO, L.S. Incidência de doença e necessidade de controle em cultivo protegido de videira. Revista Brasileira de Fruticultura, Jaboticabal, v.29, n.3, p.477-482, 2007.

COMIRAN, F.; BERGAMASCHI, H.; HECKLER, B.M.M.; SANTOS, H.P.; ALBA, D.; SARETTA, E. Microclima e produção de videiras 'Niágara Rosada' em cultivo orgânico sob cobertura plástica. Revista Brasileira de Fruticultura, Jaboticabal, v.34, n.1, p.152-159, 2012.
CONCEIÇÃO, M.A.F.; MARIN, F.R. Condições microclimáticas em um parreiral irrigado coberto com tela plástica. Revista Brasileira de Fruticultura, Jaboticabal, v.31, n.2, p.423-431, 2009.

FERREIRA, M.A.; PEDRO JÚNIOR, M.J.; SANTOS, A.O.; HERNANDES, J.L. Modificação parcial do ambiente de cultivo da videira 'Cabernet Sauvignon' sobre diferentes porta-enxertos: efeito sobre a produção e teor de sólidos solúveis, Bragantia, Campinas, v.63, n.3, p.439-145, 2004

LULU, J.; PEDRO JÚNIOR, M.J. Microclima de vinhedos cultivados sob cobertura plástica e a céu aberto. Revista Brasileira de Agrometeorologia, Santa Maria, v.14, p.106-115, 2006.

MOTA, C.S.; AMARANTE, C.V.T.; SANTOS, H.P.; ZANARDI, O.Z. Comportamento vegetativo e produtivo de videiras 'Cabernet Sauvignon' cultivadas sob cobertura plástica. Revista Brasileira de Fruticultura, Jaboticabal, v.30, n.1, p 148-153, 2008.

PEDRO JÚNIOR, M.J.; HERNANDES, J.L.; ROLIM, G.S. Sistema de condução em Y com e sem cobertura plástica: microclima, produção, qualidade do cacho e ocorrência de doenças fúngicas na videira 'Niagara Rosada'. Bragantia, Campinas, v.70, n.1, p. 228-233, 2011.

PEDRO JÚNIOR, M.J.; HERNANDES, J.L.; TECCHIO, M.A.; PEZZOPANE, J.R.M. Influencia do sistema de condução no microclima, produtividade e qualidade de cachos da videira Niagara Rosada, em Jundiaí (SP). Revista Brasileira de Fruticultura, Jaboticabal, v. 29, p. 313-317, 2007.

PEDRO JÚNIOR, M. J.; PEZZOPANE, J. R. M.; HERNANDES, J. L.; ABRAMIDES, P. L. G. Sistemas de condução da videira 'Niagara Rosada': efeitos na transmissão de radiação solar e na produtividade. Revista Brasileira de Agrometeorologia, Santa Maria, v. 14, p. 1-9, 2006. 